\title{
SAFETY AND BENEFIT OF SIMULTANEOUS BILATERAL CATARACT SURGERY IN TERTIARY CARE CENTRE
}

H. .N Sowbhagya, Pooja Patil, N. V. V. Himamshu, Kiran Kumar L, Aisha Rafi, Kirthi Raj, Liji P, Sushruth. A.

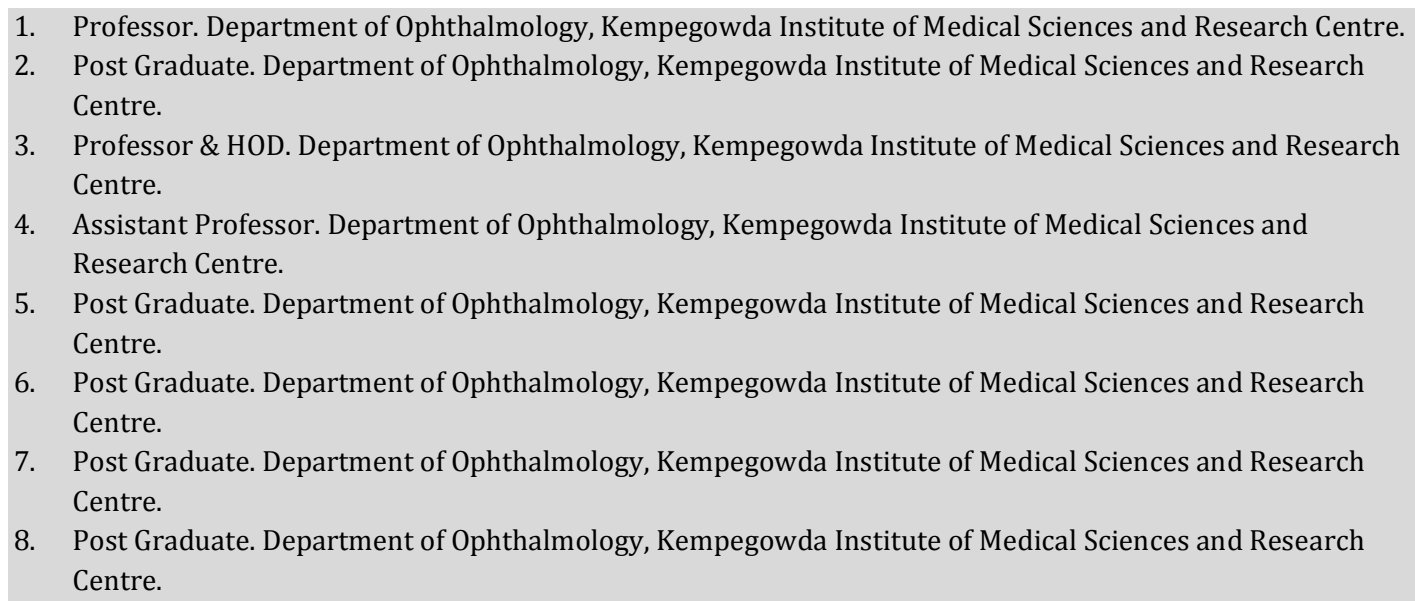

\section{CORRESPONDING AUTHOR:}

Dr. H. N. Sowbhagya,

No 41-42/45 Sri Guru Krupa,

$7^{\text {th }}$ Cross, Saraswathipuram,

Nandini Layout Main Road,

Bangalore- 560 096,

E-mail: drhnsowbhagyaappaji@gmail.com

ABSTARCT: AIM: To study safety and benefits of bilateral cataract surgery in a tertiary care ophthalmic centre of multi-specialty hospital in bilateral cataract cases DESIGN: Prospective study. MATERIALS AND METHODS: Prospective study done during May 2011 to Feb. 2012. A total 166 subjects underwent Bilateral Small Incision Cataract Surgery (SICS). Out of 166 subjects 83 subjects underwent Immediate Simultaneous Sequential Bilateral Cataract Surgery (ISBCS) in the same sitting, 83 subjects underwent bilateral cataract surgery at the interval of 48 hours Delayed simultaneous bilateral cataract surgery (DSBCS). RESULTS: All had uneventful convalescence, single hospital admission and simultaneous Bilateral Visual recovery. CONCLUSION: No case had post-operative sight threatening infections. Subjective motivation for ISBCS better than DSBCS as it was single OT visit and one time surgery

KEYWORDS: Bilateral, simultaneous, immediate simultaneous bilateral cataract surgery. Delayed simultaneous bilateral cataract surgery.

INTRODUCTION: Globally, the number of people of all ages visually impaired is estimated to be 285 million, of whom 39 million are blind. People 50 years and older represent $65 \%$ and $82 \%$ of visually impaired and blind, respectively. The major causes of visual impairment are uncorrected refractive errors (43\%) followed by cataract (33\%). ${ }^{1}$. Blindness due to cataract often bilateral presents an enormous problem in India not only in terms of human morbidity but also in terms of economic loss and social burden. The main causes of blindness in India are as follows: - Cataract (62.60\%) Refractive Error (19.70\%) Corneal Blindness (0.90\%), Glaucoma 
(5.80\%), Surgical Complications (1.20\%) Posterior Capsular Opacification (0.90\%) Posterior Segment Disorder (4.70\%) and Others (4.19\%). The estimated national prevalence of childhood blindness /low vision is 0.80 per thousand. ${ }^{2}$

Most of the cataract blinds in the country are in the rural areas while the surgical service delivery network is concentrated in the urban areas. Thus a large proportion of patients in the rural areas continue to remain blind. ${ }^{3}$ To eliminate cataract blindness in India, an estimated 9 million good-quality cataract surgeries are needed every year during 2001-2005, increasing to over 14 million surgeries needed every year during 2016-2020 on persons most likely to go blind from cataract. (4)

When standard Extra Capsular Cataract Extraction (ECCE) was the most popular procedure, patients often waited for up to several months between their first and second cataract surgeries. Large incisions were associated with a low, but significant; incidence of early wound problems, secondary infection (endophthalmitis and panophthalmitis) and suture induced astigmatism often resulted in poor uncorrected vision for 1-2 months. Visual recovery was more often delayed by prolonged corneal edema or clinically significant cystoid macular edema. Refractions often took months to stabilize.(5)

During the past decade, advances in techniques and technology (laminar air flow operation theatres, Automated UV operation theatre sterilization, Automated fumigator systems, and ultrasound instrument cleaning system and flash autoclaves have made surgical environment bio free and increases the quantity of sterile units available for the procedures instantaneously) have led to major changes in cataract surgical practice patterns. The complete transition from large incision extra capsular cataract extraction (ECCE) to phacoemulsification was driven by the ability to accelerate the visual and physical rehabilitation of cataract patients. The subsequent innovations of foldable intraocular lenses (IOLs) and small, clear corneal incisions, topical anesthesia, suture-less surgery has eliminated patching of the eyes for days and physical restrictions. In this progression towards ever faster rehabilitation, simultaneous bilateral cataract surgery (SBCS) may be the next and ultimate step in cataract surgery. (5)

A number of authors have reported their results with simultaneous bilateral cataract surgery. ${ }^{(6-7)}$ Each of these series demonstrated excellent clinical outcomes comparable to single eye cataract surgery.

From a logistical standpoint, ISBCS significantly reduces the time spent by patients and medical staff in the overall process. There are fewer postoperative visits, reduced administrative paperwork, less temporary disability and missed work, and reduced reliance on the support of family or friends. In this regard, the true economic savings of ISBCS extend beyond the cost of the second surgical facility fee. With all of these potential advantages, the critical question to ask is to what degree are the visual and refractive outcomes compromised by this practice. It is only through outcome studies such as these that this question can be critically understood and analyzed. (8)

Simultaneous bilateral ocular surgery of any kind cannot be considered if there is either a high complication rate, or if it causes a prolonged period of visual disability. However, as we have seen with LASIK, simultaneous surgery is a viable option if the complication rate is low and if vision recovery is rapid. What has changed over time, of course, is that cataract surgery can now approach LASIK with regard to safety and rapidity of recovery. (5)

The potential advantages Of ISBCS are significant. There are special circumstances, such as transportation problems or the need for general anesthesia, where subsequent second eye surgery might not otherwise be possible. With ISBCS, patients with high hyperopia or myopia 
would be spared the frustration of considerable interim anisometropia. By focusing on clinical outcomes, we physicians can understand the reduction in emotional stress or physical hardship that patient suffers while undergoing $2^{\text {nd }}$ eye surgery and the postoperative period for certain patients. For these individuals, eliminating the second separate encounter may be particularly desirable. Finally, all patients experience a faster return to full visual function and their final refraction following uncomplicated ISBCS, when compared to two sequential procedures. ${ }^{(5)}$

In any healthcare system where limited resources may impose rationing or lengthy delays in elective surgery, ISBCS might be the best way to safely extend the benefits of cataract surgery to as many eyes as possible

The Surgeons should develop their protocol for ISBCS in detail. Most of the guidelines are common. The patients must have bilateral symptomatic cataracts, must be well informed, and must desire bilateral surgery on the same day. Patients felt to be at high risk of intra operative complications like pathological myopia, uncontrolled glaucoma, hypertension diabetes mellitus and renal compromised patients are not given this option; ISBCS surgery is not performed in the event of intra operative complications with the first eye. To the list of poor candidates for ISBCS are those at risk of early postoperative complications (for example, uveitis and poorly controlled glaucoma), delayed visual recovery (for example, patients with Fuchs' corneal dystrophy), and those at greater risk for refractive surprise (for example, post-LASIK patients). (5)

MATERIAL AND METHODS: Prospective study done during May 2011 to Feb. 2012. Study of acceptance, Safety and Benefit of Bilateral Cataract Surgery, done as a part of outreach program, in a Multispecialty Teaching Hospital under community ophthalmology, after obtaining IEC permission.

Study included screening of 800 subjects of bilateral cataract cases of which 166 cases were selected based on inclusion and exclusion criteria.

\section{INCLUSION CRITERIA:}

1) Bilateral cataract cases age ranging between 19-55 years.

2) Patient willing to undergo bilateral cataract surgery

3) Medically fit cases.

4) Reliable to co operate for bilateral cataract surgery and available for regular post operative follow up.

\section{EXCLUSION CRITERIA:}

1) Debilitated elderly patients

2) Medical problems like uncontrolled diabetes, hypertension, and asthmatics.

3) Seropositive cases

4) Poor hygienic patients

5) Patients with ocular pathology like high myopes, cases of intraocular inflammation, glaucoma, ocular surface abnormalities, lid abnormalities and corneal pathologies

6) Patient below 18 yrs and above 55 yrs.

Patient above 55 yrs and below 18 yrs were excluded from study because we were considering working class for study who are fit for surgery and available for regular follow up. All the cases underwent basic screening procedures like slit lamp examination, intra ocular pressure recording and Visual acuity testing and Systemic examination for hypertension, 
obesity, skin infections, and systemic infections. After excluding obvious clinical contraindications, willingness of suitable cases for bilateral cataract surgery recorded and enrolled for the study. Study done according to ICMR guidelines and Helsinki Guidelines. Out of 800 cases 166 were enrolled for the study. All were screened for Hematology. Biochemistry, Serology, chest X-ray, ECG. Out of 166 cases, Group 1:- 83 patients (50\%) had bilateral cataract surgery on same day one after the other immediate simultaneous bilateral cataract surgery (ISBCS) and Group 2:- 83 patients (50\%) had bilateral cataract surgery at an interval of 48 hours (DSBCS). All the cases underwent Small incision sutureless cataract surgery (SISCS) under Peribulbar anesthesia. Two surgeons operated in both the groups. Cases of ISBCS received anesthesia for both eyes before surgery. A preoperative dose of Ciprofloxacin $500 \mathrm{mg}$ and alprazolam $5 \mathrm{mg}$ was given orally. All eyes were operated as individual patients, using separate cataract set, IV sets, drapes, visco-elastics, gloves and blades for each eye. However operating surgeon was the same for that particular patient. There was around 5 minutes interval between operating $1^{\text {st }}$ and $2^{\text {nd }}$ eye during which patient was painted and draped and $2^{\text {nd }}$ eye prepared for surgery. All the patients received subconjunctival betamethasone $4 \mathrm{mg}$ and gentamycin $20 \mathrm{mg}$ at the end of the surgery. Eyes were patched for 2 hours after surgery, after which protective goggles were given. Topical loteprednol etabonate $(0.5 \%)$ and tobramycin $(0.3 \%)$ and flurbiprofen $(0.03 \%)$ eye drops applied $6^{\text {th }}$ hourly for 6 weeks.

The post operative follow up was done on the first day, first week, $2^{\text {nd }}$ week, $3^{\text {rd }}$ week and $6^{\text {th }}$ week after surgery. Patients were observed on slit lamp for corneal clarity, conjunctival congestion, anterior chamber details, pupil and pseudophakic status. Visual acuity, fundoscopy and intraocular pressure (I-care rebound Tonometer) were recorded in each visit. All patients were brought to the hospital for review from screening centre for follow up. After 6 weeks of follow-up final refraction was done and spectacle given.

RESULTS: Total of 166 cases with bilateral cataract underwent bilateral cataract surgery. The median age was $53.52 \pm 2.25$ years ranged between 19 to 55 years, females $92(55.42 \%)$ and males $74(44.58 \%)$. All the cases had bilateral cataract, visual acuity ranged between hand movements to counting fingers.

In the Group-1, patients underwent ISBCS in the same sitting were comfortable and easily accepted second eye surgery. 3 out of 83 patients showed mild uveitis in one eye only, which resolved within 3 days with routine post operative treatment with addition of homatropine sulfate (2\%) two times daily for three days. They were kept admitted till the resolution and discharged. One case had unilateral corneal edema, which got resolved in three weeks. One case developed increased IOP in one eye on $2^{\text {nd }}$ post operative day ( $40 \mathrm{~mm} \mathrm{Hg}$ ) which persisted till the end of first week. It was diagnosed glaucoma secondary to viscoelastic and treated with loteprednol etabonate $(0.5 \%)$, flurbiprofen $(0.03 \%)$ eye drops and timolol maleate $(0.5 \%)$ thrice a day. IOP become normal after 10 days. Total inpatient stay was 3 days except in patients who had uveitis and increase in IOP. [Table-1]

In the Group-2, DSBCS the second eye cataract surgery was done 48 hours after the first eye surgery. The patient's primary motivation may be functional, emotional, and logistical because 4 of these patients became more apprehensive at the time of second eye surgery. As the first eye surgery was uncomplicated, visual recovery was good; they overcome the apprehension after looking at the other patient who underwent the second eye surgery from that group. In the second group one case had bulge in the anterior segment during second eye surgery and difficult to do SISCS. It was converted to Ab-externo procedure and required 
anterior vitrectomy. The hospital stay for second group was 4 days. No cases had operative or postoperative vision threatening bilateral complications.

All the patients had good visual outcome. Best Corrected Visual acuity post operative in group $16 / 6(48.1 \%), 6 / 9(37.95 \%),(6.02 \%)$ had 6/12 vision and one eye of one patient had vision less than $6 / 60$ compared to group 2 which was $6 / 6(54.81 \%), 6 / 9$ (37.95\%), 6/12 $(96.02 \%)$ and $6 / 60$ or less (0.6\%) [Table-2]

DISCUSSION: Since non-simultaneous bilateral cataract surgeries are often performed several weeks apart, the incidence of late postoperative complications, such as retinal detachment or late corneal decompensation, is less relevant to the SBCS question. Such complications would not have affected the decision or timing for second eye surgery. Likewise, intra-operative complications, such as vitreous loss, dropped nucleus, or choroidal hemorrhage, should automatically disqualify the second eye for same day surgery. Thus, in analyzing the downside to SBCS, the most important complications to consider would be those occurring during the early postoperative period. Some, such as endophthalmitis are vision threatening and others such as refractive surprise, and some, such as moderate corneal edema or toxic anterior segment syndrome, are temporary. All of these complications could have altered the timing or outcome of the second operation. Simultaneous Bilateral cataract surgery was safe and rewarding to developing countries, when performed at well structured OT and good supervision facility available.

Growing waiting lists for cataract surgery are common in many countries. Given the state of modern cataract surgery, simultaneous bilateral cataract surgery would seem to be a logical option for experienced surgeons to offer to selected patients. The patient's primary motivation may be functional, emotional, logistical, or economic. The collective literature from the past 7 years seems to support the safety of this approach when strict, conservative protocols are followed. In any healthcare system where limited resources may impose rationing or lengthy delays in elective surgery, SBCS might be the best way to safely extend the benefits of cataract surgery to as many eyes as Possible.

In ophthalmology, cost-effectiveness studies of vitamin A supplementation (9), cataract surgery, ${ }^{(10,11)}$ trichiasis surgery, (12) diabetic retinopathy screening, (13) and glaucoma screening $(14,15)$ have been performed. Cataract surgery studies support the benefit of bilateral cataract surgery by saving time and expenditure, increasing convenience for the patient, requiring fewer office visits, less manipulation of systemic medications, 1 scheduled surgical time, quicker recovery of binocular vision and return to normal life. This faster recovery yields a benefit to society (8). Bilateral cataract surgery also resulted in improvements in both binocular and monocular visual acuity, contrast sensitivity and BAT glare sensitivity. (16)

This study suggests that ISBCS is at least as safe as having surgery on one eye at a time. (Generally the rate of endophthalmitis quoted is around 1 in 1000 cases) ${ }^{17}$. The mean betterseeing eye visual acuity was significantly better (by 2 lines) in second and bilateral surgery patients than in first-eye surgery patients. ${ }^{(17)}$

In 2001, Smith and Liu reviewed the literature citing seven reports published between 1995-8 with a total of 2859 SBCS patients (18). Some series utilized ECCE while others used phaco procedures. There was a combined total of four cases of endophthalmitis, none bilateral, equaling a rate of $0.14 \%$. Since then four additional series, including that of Johansson and Lundh, have been published demonstrating excellent results(19) Totan's series included results in 19 pediatric or teenaged patients. ${ }^{(20)}$ Kontkanen and Kontkanen recently published results from 2715 patients operated on by two surgeons between 1996 and 2001.(21), there were two 
cases of unilateral endophthalmitis, none bilateral, in this series. Thus, although the risk of bilateral endophthalmitis is often cited as a prime deterrent to SBCS, there has not been a single occurrence in the 6000 cases reported in the literature since 1995.

Short of adjustable IOL technology, current surgical techniques would seem to have improved the feasibility of SBCS when compared to the past. Biometry accuracy has been improved with the use of immersion ultrasound or optical coherence biometry (IOL Master, Zeiss Humphrey). The growing preference of combining topical non-steroidal anti-inflammatory drugs with steroids postoperatively may further reduce the incidence of cystoid macular edema (CME). The reduction in average phaco times achieved through newer phaco technology and methods such as phaco chop are diminishing the incidence of early corneal edema. Finally, small, temporal, clear corneal incisions are astigmatically neutral and stabilize quickly. Johansson and Lundh state that a prerequisite for SBCS is the patient's informed preference for this. An additional prerequisite should be the surgeon's own confidence and ability to consistently accomplish these goals of refractive accuracy, a low complication rate, and rapid visual recovery without patch.

CONCLUSION: There was not much difference between immediate simultaneous bilateral cataract surgery at the same sitting and Delayed simultaneous bilateral cataract surgery in this study. Subjective acceptance of the patients was better in the ISBCS than DSBCS because

1) Emotional burden of preparing for the second operation was not present.

2) This also minimized the apprehension of patients which occurred for the second cataract surgery in this study.

3) Second operation theatre preparation for the DSBCS was not necessary in the ISBCS

Sequential Bilateral Cataract Surgery of either category can be taken up for bilateral cataract patients in an ophthalmic setup with sufficient infrastructure, like ophthalmic institutes and Multispecialty teaching hospitals. Thus we can minimize the rates of operative and postoperative complications as compared to peripheral make shift operation theatres (Camp OTs). A good option for outreach programs for selected and patients willing for bilateral cataract surgery especially in developing countries like India where transportation, poverty, dependency predominates. This will prevent downtrodden dependent aged patients coming with ocular emergencies like lens induced glaucoma, phacolytic uveitis. Auto couching, phacoanaphylactic uveitis and other ocular complications of the hypermature cataract which is a common scenario in our country. This will also reduce the burden of blindness due to cataracts and related morbidity.

ACKNOWLEDGEMENT: Department of ophthalmology sincerely thanks

1. IEC committee for giving IEC clearance

2. Institute for providing the entire infrastructure necessary for the study.

\section{REFERENCE:}

1. Donatella Pascolini, Silvio Paolo Mariotti Br J Ophthalmol 2012;96:614-618

2. Ref Community Eye Health J Indian Supplement 2008; 21(65): s103-s104

3. Angra SK, Murthy GV, Gupta SK, Angra V Indian J Med Res. 1997 Oct;106:312-24.

4. Clin Experiment Ophthalmol. 2003 Feb;31(1):4-5

5. D F Chang. Br J Ophthalmol. 2003 March; 87: 253-254.) 
6. Benezra D, Chirambo MC. Bilateral versus unilateral cataract extraction: advantages and complications. Br J Ophthalmol 1978; 62:770-3.

7. Sharma TK, Worstmann T. Simultaneous bilateral cataract extraction. J Cataract Refract Surg 2001; 27:741-4.

8. Simultaneous bilateral cataract surgery: Financial differences among nations and jurisdictions Steve A. Arshinoff, MD, Sylvia H. Chen J CATARACT REFRACT SURG - VOL 32, AUGUST 2006

9. http://bmj-bjo.highwire.org/content/83/3/343.full.pdf+htm Bobadilla J-L, Cowley P, Musgrove $\mathrm{P}$, et al. Design, content and financing of essential national packages of health services. In: Murray CJL, Lopez AD, eds, Global Comparative Assessments In the Health Sector: Disease Burden, Expenditures and Intervention Packages. Geneva, Switzerland, World Health Organization, 1993; 171-180, 1994 171-180. Also in: Bull World Health Org 1994; 72: 653-662

10. Murthy GV, Sharma P. Cost analysis of eye camps and camp-based cataract surgery. Natl Med J India 1994; 7:111-114

11. Evans TG, Ranson MK, Kyaw TA, Ko CK. Cost effectiveness and cost utility of preventing trachomatous visual impairment: lessons from 30 years of trachoma control in Burma. Br J Ophthalmol 1996; 80: 880-889

12. Javitt JC, Aiello LP. Cost-effectiveness of detecting and treating diabetic retinopathy. Ann Intern Med 1996; 124:164-169

13. Boivin JF, McGregor M, Archer C. Cost effectiveness of screening for primary open angle glaucoma. J Med Screen 1996; 3:154-163

14. Tuck MW, Crick RP. The cost-effectiveness of various modes of screening for primary open angle glaucoma. Ophthalmic Epidemiol 1997; 4:3-17

15. Elliott DB, Patla A, Bullimore MA. Improvements in clinical and functional vision and perceived visual disability after first and second eye surgery. Br J Ophthalmol 1997; 81:889-95.

16. Steve A. Arshinoff, MD, FRCSC Paul A. Bastianelli, BSc Journal of Cataract \& Refractive Surgery

Volume 37, Issue 12 , Pages 2105-2114, December 2011

17. Vijaya K. Gothwal, PhD, Thomas A. Wright, BPsych(Hons), Ecosse L. Lamoureux, PhD, Jyoti Khadka, PhD, Colm McAlinden, PhD, Konrad Pesudovs, PhD Journal of Cataract \& Refractive Surgery, Vol. 37, No. 7. (July 2011), pp. 1208-1216

18. Smith GT, Liu CSC. Is it time for a new attitude to "simultaneous" bilateral cataract surgery? Br J Ophthalmol 2001;85:1489-96

19. BA Johansson, B L Lundh Br J Ophthalmol 2003;87:285-290 doi:10.1136/bjo.87.3.285

20. Totan Y, Bayramlar H, Cekic O, et al. Bilateral cataract surgery in adult and pediatric patients in a single session. J Cataract Refract Surg 2000;26:1008-11

21. Sarikkola AU, Kontkanen M, Kivelä T, Laatikainen L J Cataract Refract Surg. 2004 Jun;30(6):1335-41. 

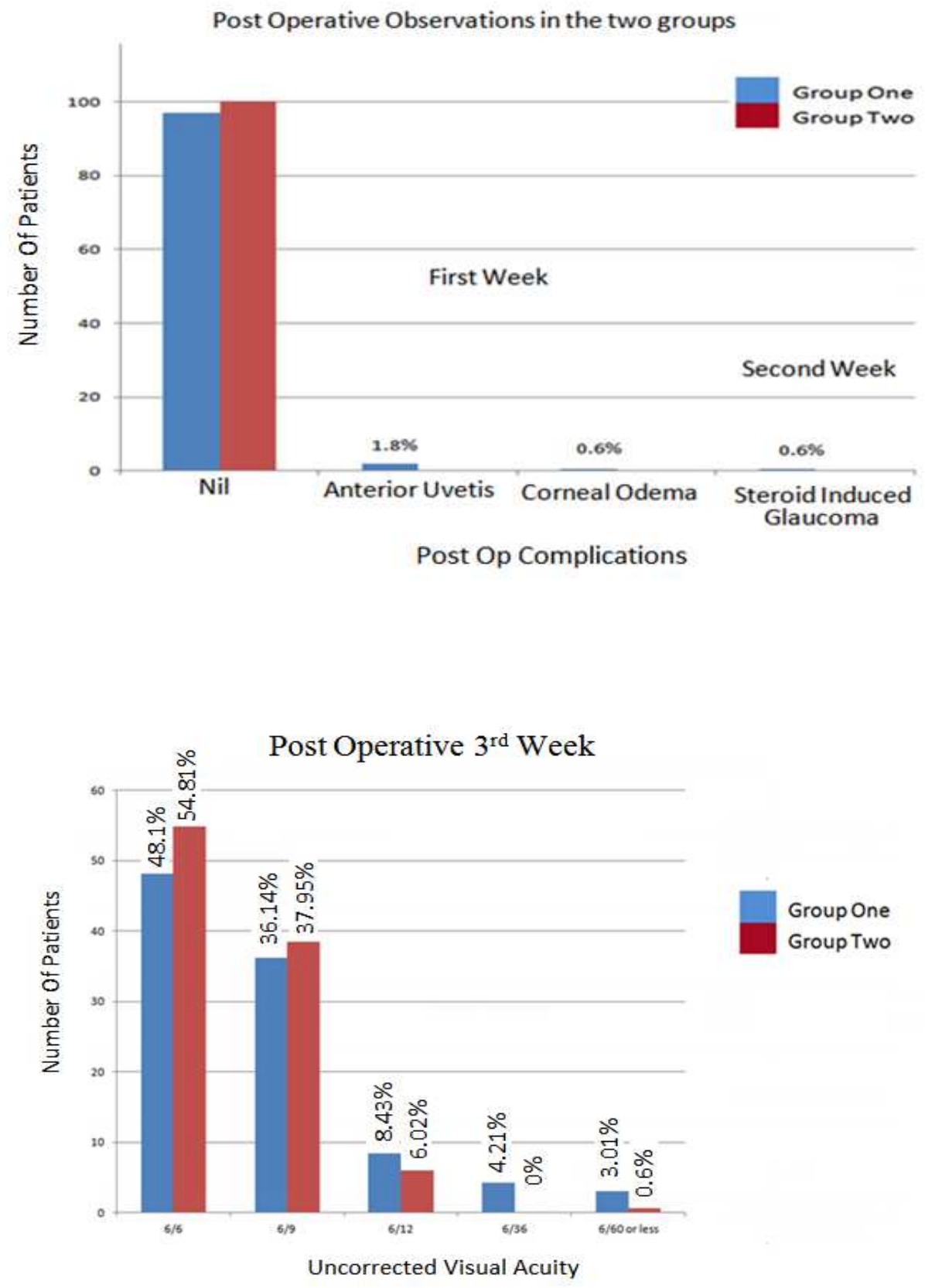